\title{
Improving hydride conductivity in layered perovskites via crystal engineering
}

\author{
Harry W. T. Morgan, ${ }^{*} \dagger$ Harry J. Stroud, ${ }^{\ddagger}$ and Neil L. Allan ${ }^{\ddagger}$ \\ $\dagger$ Department of Chemistry, University of Oxford, South Parks Road, Oxford, OX1 3QR \\ $\ddagger$ School of Chemistry, University of Bristol, Cantock’s Close, Bristol, BS8 1TS \\ E-mail: harry.morgan@chem.ox.ac.uk
}

\begin{abstract}
Hydride ion conduction in layered perovskites is of great interest for sustainableenergy applications. In this report we study $\mathrm{Ba}_{2} \mathrm{ScHO}_{3}$, a recently synthesized oxyhydride with an unusual anion ordering, using a multifaceted density functional theory approach involving both transition state calculations and molecular dynamics simulations. Beyond simply identifying the key ion migration pathways, we perform detailed analysis of transition states and identify key interactions which drive trends in ionic mobility. Our key findings are that ionic mobility is, remarkably, independent of hydride-oxide disorder, the dominant migration pathway changes under pressure, and a reduction in A-site cation size accelerates hydride diffusion. Local structural flexibility along migration pathways is understood in terms of dimensionality and ionic size, and we thus identify crystal engineering principles for rational design of ion conductors. On the basis of our new insights into these materials, we predict that $\mathrm{Sr}_{2} \mathrm{ScHO}_{3}$ will show improved conductivity over existing analogues.
\end{abstract}




\section{Introduction}

Ion conductors are essential materials for fuel cells and will become ever more important as more sustainable fuel technologies are adopted. Hydrogen has the potential to play a key role in a "green" energy economy and is therefore the subject of much current research addressing efficient production, storage and use. Proton conduction has been known for some decades in ceramic, organic and polymeric materials. ${ }^{1-3}$ By contrast, hydride ion conductivity is a much more recent and less thoroughly investigated phenomenon, confined to a few metal hydrides and oxyhydrides. ${ }^{4-7}$

Oxide anion conduction in mixed metal oxides has received enormous attention due to its applicability to solid oxide fuel cells $(\mathrm{SOFCs})^{8}$ and is likely to be related to hydride conduction. High oxide ion mobility is observed structure types ranging from binary materials with fluorite structures such as $\mathrm{Bi}_{2} \mathrm{O}_{3}$ and $\mathrm{ZrO}_{2}-\mathrm{Y}_{2} \mathrm{O}_{3}$ to complex materials based on perovskite $\left(\mathrm{ABO}_{3}\right)$ or apatite $\left(\mathrm{M}_{10}\left(\mathrm{XO}_{4}\right)_{6} \mathrm{O}_{2+y}\right)$ frameworks. The great advantage of perovskites for optimizing device performance is their chemical flexibility; their structural and transport properties can be subtlely controlled by doping on any anion or cation site. As well as $\mathrm{ABO}_{3}$ cubic perovskites, the family also includes reduced derivatives such as the $\mathrm{A}_{2} \mathrm{~B}_{2} \mathrm{O}_{5}$ brownmillerites and layered intergrowth structures such as the $\mathrm{A}_{n+1} \mathrm{~B}_{n} \mathrm{O}_{3 n+1}$ Ruddlesden-Popper phases, shown in Figure 1. This final class has been found to be particularly useful for cathode materials, for which mixed electronic and ionic conduction is required. ${ }^{8}$ In $\mathrm{La}_{2} \mathrm{NiO}_{4+x}$ the interstitial oxide anions have exceptionally high diffusivity through the "rock salt" layers of the structure. ${ }^{9}$

In the last five years, layered perovskite oxyhydrides have emerged as a particularly impor-

tant class of hydride conductors following the synthesis of the $\mathrm{La}_{2-x-y} \mathrm{Sr}_{x+y} \mathrm{LiH}_{1-x+y} \mathrm{O}_{3-y}$ series of $n=1$ Ruddlesden-Popper (RP) phases by Kobayashi ${ }^{7} .{ }^{10}$ This structure type consists of single layers of octahedra, called "perovskite" layers, separated by "rock salt" layers, as indicated in Figure 1(c), so called due to the local coordination environments. In most known RP hydride conductors the hydride ions are preferentially located in the perovskite layers, 


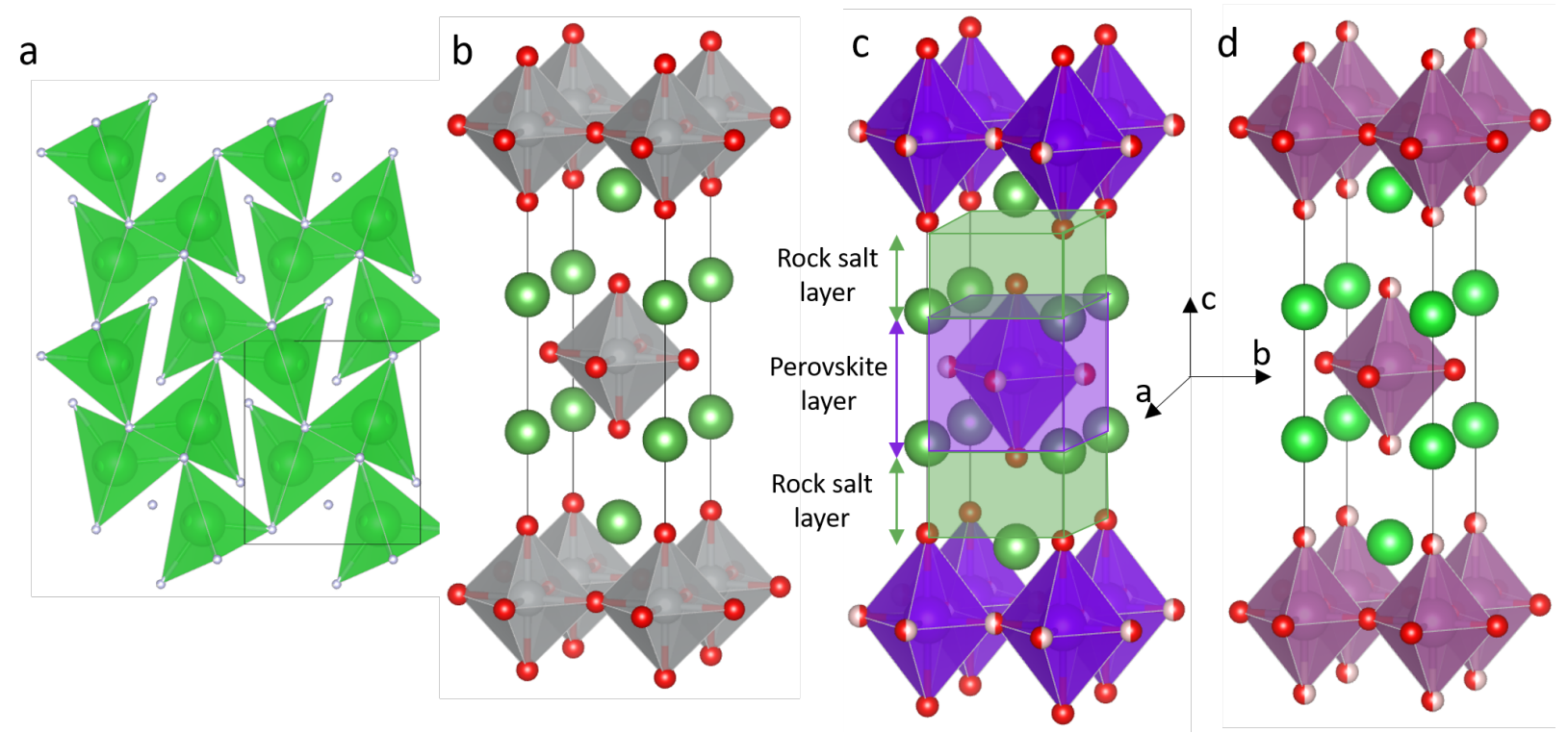

Figure 1: Selected anion conductors (a) $\mathrm{BaH}_{2}$ viewed along the $b$ axis (b) $\mathrm{La}_{2} \mathrm{NiO}_{4}$ (c) $\mathrm{La}_{2} \mathrm{LiHO}_{3}$; (d) $\mathrm{Ba}_{2} \mathrm{ScHO}_{3}$. Light green spheres represent barium, dark green lanthanum, grey nickel, purple lithium, lilac scandium, red oxygen, and white hydrogen. Mixed red/white spheres refer to oxide/hydride disorder. The set of axes refers to cells (b)-(d).

as shown in Figure 1(c), and thus interact principally with the B-site cations - this is the case for the above La-Sr-Li system as well as for phases containing $d^{n}$ transition metal ions such as $\mathrm{LaSrCoO}_{3} \mathrm{H}_{0.7}{ }^{11,12}$ and $\mathrm{Sr}_{2} \mathrm{VO}_{3} \mathrm{H} .{ }^{13}$ Bai et al. ${ }^{14}$ have investigated hydride diffusion in the $\mathrm{La}_{2-x-y} \mathrm{Sr}_{x+y} \mathrm{LiH}_{1-x+y} \mathrm{O}_{3-y}$ family using ab initio molecular dynamics (AIMD), finding that vacancies played a key role in diffusion of $\mathrm{H}^{-}$in the perovskite layer. Additionally, Fjellvåg et al. ${ }^{15}$ have studied the $\mathrm{La}_{2} \mathrm{LiHO}_{3}$ member of that series and found hydride mobility within the perovskite layer to be more facile than in the rock salt layer, which they link to La-O covalency, and that vacancies promote hydride diffusion. ${ }^{16}$

In 2019 Takeiri et al. ${ }^{17}$ reported the high-pressure synthesis of $\mathrm{Ba}_{2} \mathrm{ScHO}_{3}$ and observed hydride conduction at $300{ }^{\circ} \mathrm{C}$. This compound also adopts the $n=1 \mathrm{RP}$ structure but differs from previous examples in that the hydrides are distributed over the "apical" points of the octahedra and so reside in the rock salt layer, as indicated in Figure 1(d). This difference can be explained by straightforward electrostatic considerations, whereby the most positively charged cations coordinate to the most negatively charged cations. Placing the mobile hy- 
drides on these sites may enable hydride conduction through the rock salt layer, perhaps via interstitial sites inaccessible in other hydride conductors, a phenomenon observed in certain oxide conductors ${ }^{9,18}$ but unknown in perovskite oxyhydrides.

In a recent preprint, Kuwubara et al. used density functional theory (DFT) to calculate the formation energies of various point defects in $\mathrm{Ba}_{2} \mathrm{ScHO}_{3}$ and investigate their mobilities. Their findings indicate that vacancy and interstitial hydride defects form more readily than the corresponding oxide defects and that the ionic conductivity arises from these hydride defects.

Here we employ DFT to identify the most important mechanisms of hydride conduction in $\mathrm{Ba}_{2} \mathrm{ScHO}_{3}$ by a combined computational approach employing both AIMD simulations and static calculations of transition state energies. Transition state geometries are analysed and trends understood in terms of crystal engineering. We also explore the effects of anion ordering, pressure, and cation substitution on anion transport in order to provide new testable predictions to guide future synthetic searches and device design involving anion conductors.

\section{Methods}

Calculations were performed using periodic plane-wave DFT implemented in VASP ${ }^{19}$ with PAW pseudopotentials ${ }^{20}$ and the PBE exchange-correlation functional. ${ }^{21}$ For static calculations, a plane-wave cutoff of $600 \mathrm{eV}$ was used. The irreducible part of the Brillouin zone of the crystallographic cell was sampled with a $7 \times 7 \times 2 \mathrm{k}$-point grid, and that of the $3 \times 3 \times 1$ supercell with a $2 \times 2 \times 2$ grid. Reaction barriers were calculated with the climbing image nudged elastic band (CI-NEB) method using the implementation in the VASP Transition State Tools (VTST), ${ }^{22,23}$ with three images used for each calculation. Lattice parameters were fixed during CI-NEB calculations. All AIMD simulations were performed on the $3 \times 3 \times 1$ supercell in the canonical (NVT) ensemble with constrained cell shapes and volumes, and a temperature of $700{ }^{\circ} \mathrm{C}$. The plane-wave energy cutoff was set to $400 \mathrm{eV}$ and k-point sam- 
pling occurred at the gamma point only. Simulations were performed for 100 ps with a 1 fs timestep, and data were collected after equilibration periods of up to 6 ps. Temperature was controlled using the Nosé-Hoover thermostat. ${ }^{24,25}$ Static calculations were visualized with VESTA, ${ }^{26}$ and simulations with VMD. ${ }^{27}$

\section{Results}

\section{Hydride conduction in $\mathrm{Ba}_{2} \mathrm{ScHO}_{3}$}

We optimized the geometries of different possible unit cells of $\mathrm{Ba}_{2} \mathrm{ScO}_{3} \mathrm{H}$ containing 2 formula units, each with a different anion ordering as shown in Figure S3, since the compound contains oxide/hydride disorder over the apical sites. Our results for the relative energies of anion orderings agree with previous calculations ${ }^{17}$ and so we focus on the lowest energy arrangement, which contains alternating all-hydride and all-oxide rock salt layers. The cell parameters and atomic positions were optimized, and the resulting lattice parameters $a$, $b, c$ are $4.184 \AA, 4.178 \AA$ and $13.046 \AA$ respectively. These are in good agreement with experimental values at $300{ }^{\circ} \mathrm{C}$ of $4.18 \AA(a, b)$ and $13.7 \AA(c)$; our calculations are in the static limit, i.e. $0 \mathrm{~K}$ and without lattice vibrations, and orthorhombic distortions at low temperature are common in Ruddlesden-Popper phases. ${ }^{7}$

We have identified four possible movements of hydride ions through the rock salt layer which account for the roles of interstitial and vacancy defects, the formation of which have been shown to be facile. Defective cells were modelled using a $3 \times 3 \times 1$ supercell, and charge neutrality was maintained by means of a compensating background charge. We have confirmed that this background charge has no significant effect on the results - details can be found in Figure $\mathrm{S} 2 . \mathrm{Ba}_{2} \mathrm{ScHO}_{3}$ has been shown experimentally to have apical sites occupied by disordered hydride and oxide ions in a 1:1 ratio. As discussed above we chose to model $\mathrm{H}^{-}$ migration in a cell with one rock salt layer entirely occupied by hydride anions and the other entirely occupied by oxide anions, but we find that our results are not strongly dependent 
on the choice of anion ordering pattern (vide infra).

The migration pathways, CI-NEB barrier heights and transition states are shown in Figure 2. The possible hops involving the interstitial defect involve both intersticialcy and interstitial mechanisms. The former involves a knock-on displacement of a hydride on a lattice site by the interstitial ion and the latter describes direct movement of a hydride from one interstitial site to the next. The vacancy hops are termed 'Along' and 'Across' to describe their movement relative to the rock salt layer in that the former mechanism does not change the $c$ coordinate of the mobile hydride. Figure 2 shows that the lowest energy pathway is the interstitialcy hop, a correlated motion involving two hydride ions, with a barrier height of $0.35 \mathrm{eV}$. In the transition state each hydride is in approximately trigonal planar coordination, with three $\mathrm{Ba}^{2+}$ cations at $2.56 \AA$. The highest energy barrier is that for the interstitial hop, at $0.86 \mathrm{eV}$; in this transition state the mobile hydride has two $\mathrm{Ba}^{2+}$ cations at $2.36 \AA$, describing linear coordination, and four in a second coordination shell at $3.66 \AA$. There are also two $\mathrm{H}^{-}$neighbours at $2.39 \AA$ which incur significant electrostatic repulsion, rendering this transition state unfavourable.

The 'Across' and 'Along' vacancy hops are intermediate in energy, at $0.41 \mathrm{eV}$ and 0.62 $\mathrm{eV}$ respectively. In both cases, in the transition state the mobile hydride lies within a $\mathrm{Ba}$ tetrahedron but is displaced from its centre, towards a tetrahedral edge, shortening the distance travelled between sites. These displacements explain the difference in energies between the two. In the lower-energy 'Across' hop TS the hydride is displaced strongly, with H-Ba bond lengths of $2.55 \AA(\times 2), 3.03 \AA$ and $3.09 \AA$. The displacement from the centre of the tetrahedron is in the direction of another Ba cation, at $4.47 \AA$, so it offers some additional stabilization of the TS. Meanwhile, the mobile hydride in the 'Along' hop TS is located much closer to the centre of the Ba tetrahedron, with cations at $2.62 \AA$ and $2.84 \AA$, because the distortion moves it closer to an oxide anion. This pathway is therefore longer in order to reduce anion-anion repulsions and is the higher energy vacancy migration.

The mean square displacement (MSD) plots for both cells show the values for $\mathrm{H}$ climbing 

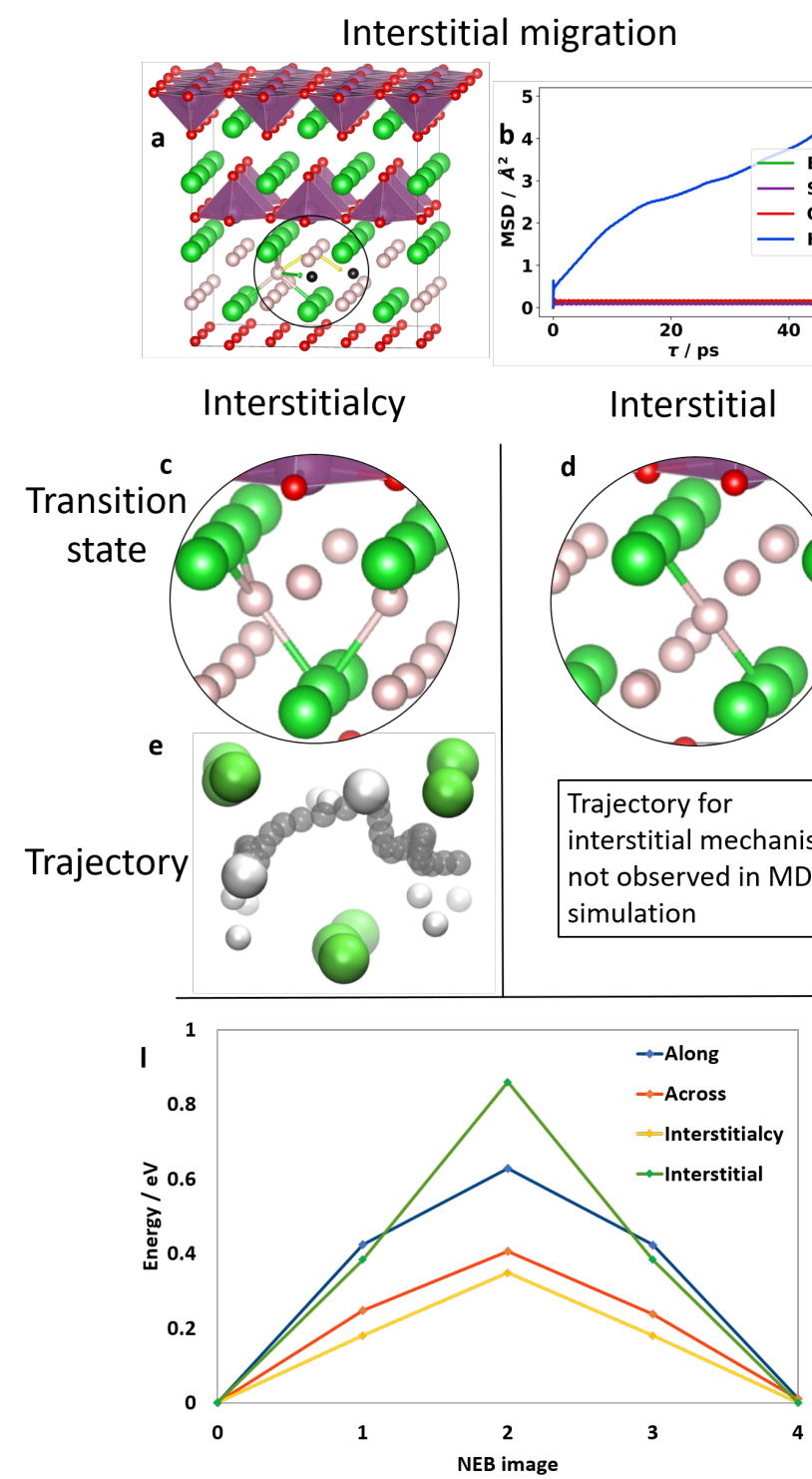

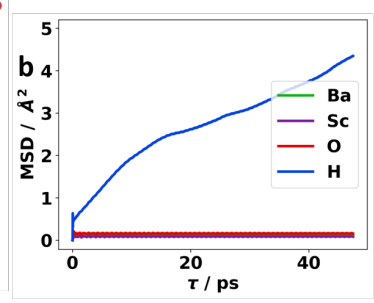

Interstitial

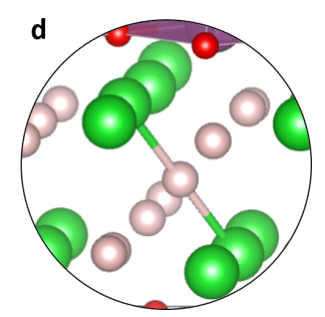

Trajectory for interstitial mechanism not observed in MD simulation
Vacancy migration

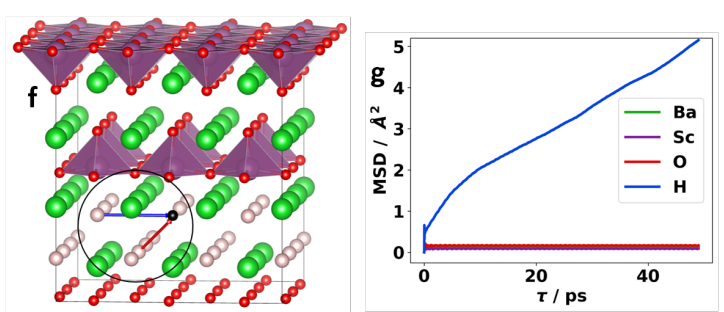

Along

Across

h

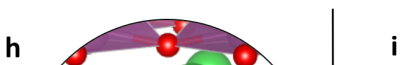

त $i$

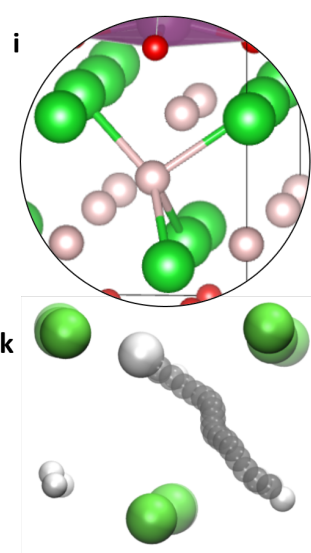

m
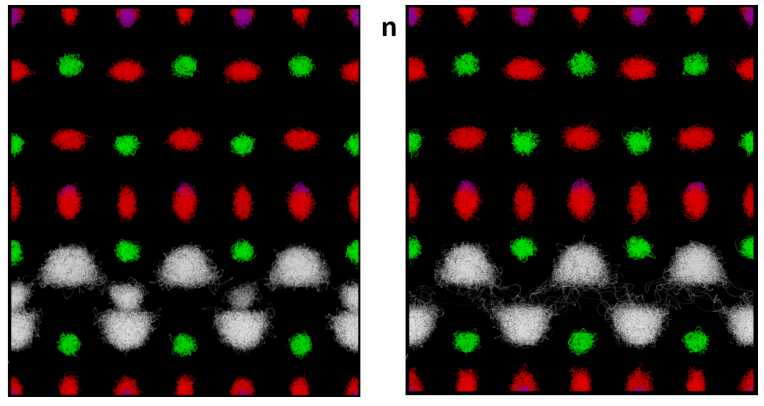

Figure 2: Summary of hydride mobility in $\mathrm{Ba}_{2} \mathrm{ScHO}_{3}$ with $\mathrm{Ba}$ in green, $\mathrm{Sc}$ in lilac, $\mathrm{H}$ in white and $\mathrm{O}$ in red. (a, f) Pathways considered for an interstitial and a vacancy defect respectively, with colour coded arrows matching the legend on graph (1). Black spheres mark the hop end points and circles highlight the parts of the cell shown in detail in parts (c, d, h, i). (b, g) MSD plots for $\mathrm{Ba}_{2} \mathrm{ScHO}_{3}$ containing an interstitial and a vacancy defect respectively. (e) Interstitialcy trajectory, viewed along $a$ with images shown every $4 \mathrm{fs}$. (j, k) 'Along' and 'Across' trajectories respectively, viewed along $a$ and $b$ respectively. (l) CI-NEB reaction barriers for all pathways. (c, d, h, i) Transition states for Interstitialcy, Interstitial, 'Along' and 'Across' hops respectively. (m, n) 100 ps trajectory of $\mathrm{Ba}_{2} \mathrm{ScHO}_{3}$ containing an interstitial and a vacancy defect respectively, viewed along $a$.

throughout the simulation, while those for all other elements oscillate around a constant value. This indicates that the hydrides are the only mobile ions in the system, all others 
vibrating about their lattice positions, as further supported by the trajectories shown in Figure 2(m,n). The hopping pathways observed in the simulation are as we expect from the calculated barriers; hydride diffusion in the interstitial cell occurs exclusively by the interstitialcy mechanism, while the vacancies travel by both mechanisms but the 'Along' mode appears more frequently. From these MSDs we have estimated diffusion constants for the interstitial and vacancy of $1.1 \times 10^{-10} \mathrm{~m}^{2} \mathrm{~s}^{-1}$ and $1.4 \times 10^{-10} \mathrm{~m}^{2} \mathrm{~s}^{-1}$ respectively. These values give us an order-of-magnitude estimate for diffusivity in $\mathrm{Ba}_{2} \mathrm{ScHO}_{3}$ and will be most informative when compared to those for other systems (vide infra).

To investigate the possibility of longer-range correlated motion involving multiple atoms, we considered a pathway of two successive interstitialcy-type displacements. The two displacements occurred on opposite sides of the rock salt layer and therefore proceeded in mutually orthogonal directions. The pathway was found to contain two barriers, both with heights equal to that of the simple two-atom interstitialcy hop, and the energy of the system returned to its minimum value in the middle of the reaction coordinate (Figure S5). This suggests that correlated motion of three atoms does not serve to provide low-energy interstitial motion pathways but does indicate that rapid successive hops of this type may be possible.

\section{Anion disorder-independence of diffusivity}

$\mathrm{Ba}_{2} \mathrm{ScHO}_{3}$ contains hydride and oxide ions distributed randomly across the rock-salt layer sites in a 1:1 ratio, but for our model we must specify an ordering. As discussed above, our calculations and simulations were performed using the lowest energy ordering in which the ions are segregated into all-hydride and all-oxide rock salt layers, so it is important to confirm that the chosen anion ordering pattern do not strongly affect the calculated hydride hopping barriers. This was investigated by generating a more disordered anion configuration, in which both rock salt layers in the cell contain oxide and hydride ions in equal number. The configuration ensures that each scandium ion is coordinated to one hydride and is shown in Figure 3(a). Barriers for all four mechanisms were calculated for this anion ordering - Figure 
3(b,c) show, respectively, the barriers for the disordered configuration and a comparison between this and the lowest energy configuration.
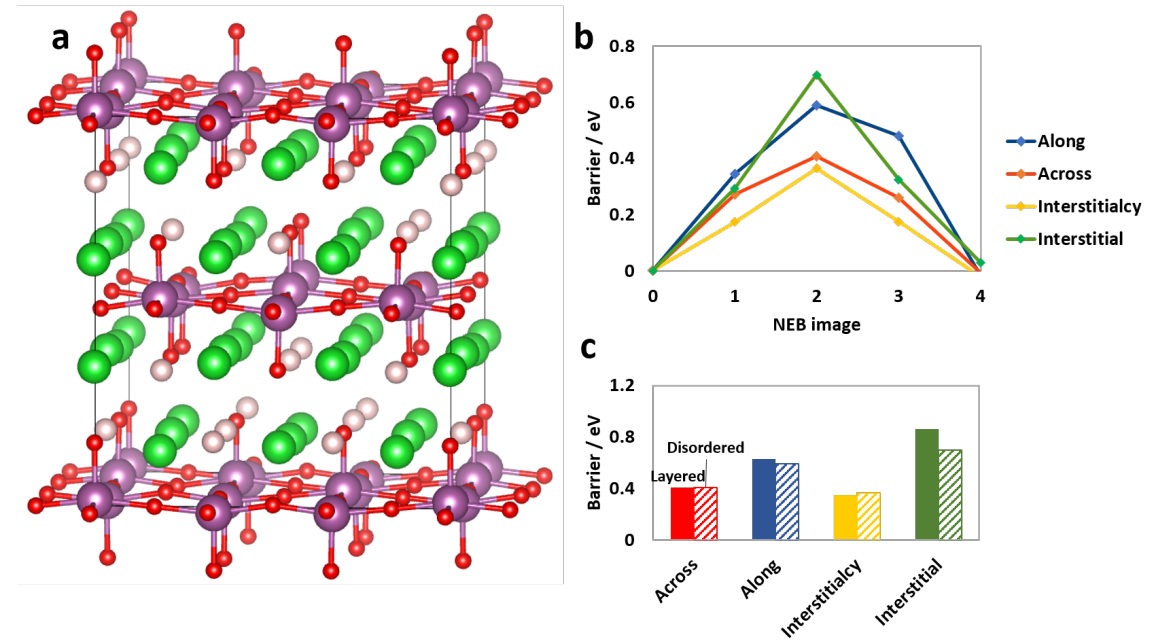

Figure 3: (a) Geometry used to test the effect of anion ordering on hydride mobility barriers (b) Activation barriers for hydride diffusion (c) comparison of barriers in the disordered configuration to those for the lowest energy layered configuration.

The barriers in Figure 3(b) show that the energetic ordering of the pathways is unchanged by the introduction of anion disorder, with interstitialcy and 'Across' vacancy migration the lowest energy mechanisms. Figure 3(c) then shows that there is very little quantitative change to the barriers, so we can conclude that anion disorder does not strongly affect hydride mobility and our treatment of it is not a significant source of error in our model.

\section{High pressure mechanism change}

Since $\mathrm{Ba}_{2} \mathrm{ScHO}_{3}$ is synthesized under high-pressure conditions it is important to establish the effects of pressure on the hydride mobility. RP phases undergo a pressure-induced phase transition, known as the " $B 1-B 2$ " 28 transition, in which the perovskite layers shift such that the ions in the inter-layer spacing change from rock-salt ordering to $\mathrm{CsCl}$ ordering. Our calculated transition pressure in the static limit is approximately $30 \mathrm{GPa}$, where the enthalpies of the low and high pressure structures are equal (Figure S6). We therefore studied hydride mobility at $20 \mathrm{GPa}$, high enough to give a clear indication of pressure-dependent 
behaviour while still well below the transition pressure.

To understand the effect of pressure on the defective cells of $\mathrm{Ba}_{2} \mathrm{ScHO}_{3}$, we carried out an analysis similar to that used for $\mathrm{Sr}_{2} \mathrm{VO}_{3} \mathrm{H}^{13}$ by dividing the cell into octahedral and rock salt blocks, as shown in Figure 1(c), and measuring their compressibilities. Vacancy and interstitial cells show the same behaviour; geometrical parameters are reported in Table S1. Crucially, the hydride-containing rock salt layer is compressed by around $14 \%$, the perovskite layer by $10 \%$ and the oxide-containing rock salt layer by $7 \%$, consistent with results for other RP oxyhydrides which show that hydride ions are twice as compressible as oxides. ${ }^{29}$ Figure 4 shows the energies of the transition states for the four pathways at $0 \mathrm{GPa}$ and 20 GPa. Pressure has a pronounced effect on the relative heights of the barriers for different motions as the mobility of vacancies falls far less dramatically than that of interstitials. The vacancy hop barriers rise by less than $50 \%$, to $0.56 \mathrm{eV}$ ('Across') and $0.78 \mathrm{eV}$ ('Along'), while the interstitial hop barriers more than double to $1.18 \mathrm{eV}$ (interstitialcy) and $1.80 \mathrm{eV}$ (interstitial). For each defect type the zero pressure orderings are preserved, with vacancies more mobile by the 'Across' mechanism and interstitial defects moving preferentially by the interstitialcy mechanism.

The MSD plots in Figure 4(c,d), for interstitial and vacancy cells respectively, show contrasting hydride behaviour to that observed at zero pressure (Figure 2(b,g)). Rather than increasing with time, the hydrogen MSDs indicate that hydride motion is vibrational rather than diffusive and thus migration is greatly hindered by compression to 20 GPa.

The transition states for all hops, shown in Figure S7, have similar coordination environments at $20 \mathrm{GPa}$ to those at ambient pressure but, unsurprisingly, compression of the rock salt layer leads to important differences in bond lengths. The interstitialcy transition state still contains trigonally coordinate mobile hydrides, with $\mathrm{H}$-Ba bonds of $2.37 \AA$, but the two anions are displaced outwards from the centre of the $\mathrm{Ba}_{3}$ triangle; the trigonal planar site, through which both hydrides must pass, contains far more severe short-range H-Ba repulsions at $20 \mathrm{GPa}$ than at zero pressure, so at $20 \mathrm{GPa}$ each hydride passes through the "local 

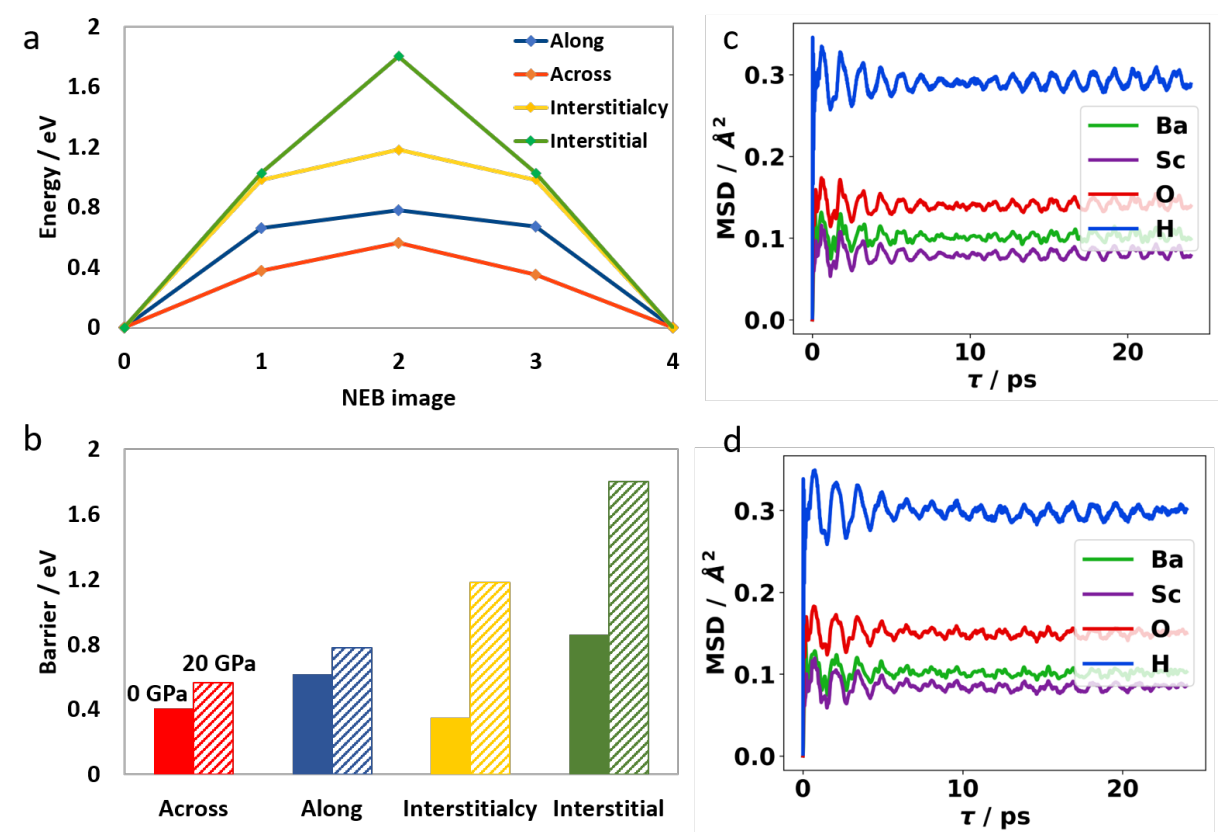

Figure 4: (a) CI-NEB hydride mobility barriers at $20 \mathrm{GPa}$. (b) Comparison of barriers at $0 \mathrm{GPa}$ (solid bars) and $20 \mathrm{GPa}$ (hatched bars). (c), (d) MSD plots for the interstitial and vacancy cells, respectively, at $20 \mathrm{GPa}$.

transition state" sequentially. This gives the TS more "di-interstitial" character, with the normal lattice site vacant, and raises the energy relative to the zero pressure case. In the 2-coordinate TS for the interstitial hop, the shortest H-Ba bond lengths are $2.18 \AA$ and the farther four are at $3.33 \AA$, with the two nearest hydrides at $2.10 \AA$. The combination of H-H electrostatic repulsions and $\mathrm{H}-\mathrm{Ba}$ Pauli repulsions once again make this the highest energy pathway.

The mobile hydride in the 'Along' vacancy hop occupies a pseudo-tetrahedral site, as before, with pairs of $\mathrm{H}$-Ba bonds at $2.39 \AA$ and $2.46 \AA$. In the lower-energy 'Across' case, the hydride is displaced in the same direction as at zero pressure but now lies much closer to the 4-coordinate site, with bond lengths of $2.36 \AA(\times 2), 2.56 \AA(\times 1)$ and $2.57 \AA(\times 1)$. This is most likely because the 2-coordinate site on the tetrahedral edge is now too small to contain $\mathrm{H}^{-}$; we were unable to find a TS at this site without significant distortions throughout the unit cell to lengthen the shortest H-Ba bonds. 


\section{Improved hydride conduction in $\mathrm{Sr}_{2} \mathrm{ScHO}_{3}$}

To search for new and improved hydride conductors, we investigated the effect of A-site cation size by replacing barium by strontium to give $\mathrm{Sr}_{2} \mathrm{ScHO}_{3}$. Searches on the Inorganic Crystal Structure Database (ICSD) indicate that this compound is as yet uncharacterized. Full geometry optimization of the unit cell gives lattice parameters in $\AA$ of $(4.099,4.084$, 13.206) - a reduction of around $2 \%$ in $a$ and $b$ and around $6 \%$ in $c$ relative to the Ba analogue.
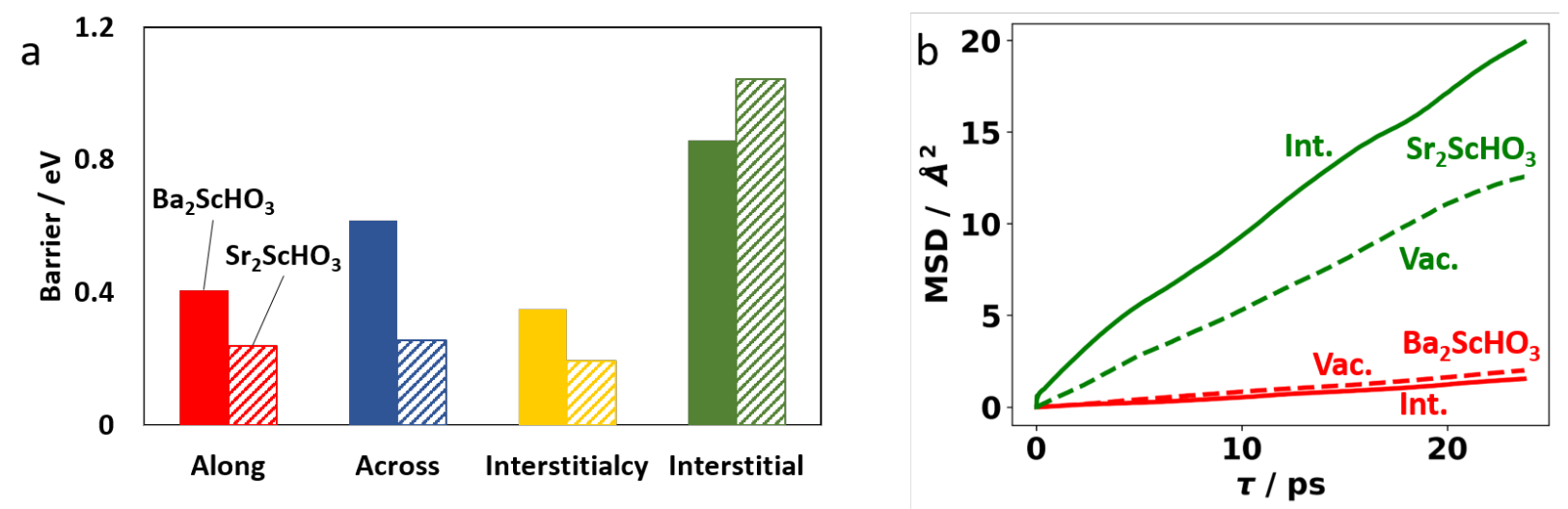

Figure 5: Comparison of hydride mobility in $\mathrm{Sr}_{2} \mathrm{ScHO}_{3}$ to $\mathrm{Ba}_{2} \mathrm{ScHO}_{3}$. (a) Comparison of barriers for equivalent processes in $\mathrm{Ba}_{2} \mathrm{ScO}_{3} \mathrm{H}$ (solid bars) and $\mathrm{Sr}_{2} \mathrm{ScHO}_{3}$ (hatched bars). (b) MSD plots for hydride ions in interstitial (solid lines) and vacancy (dashed lines) cells of $\mathrm{Sr}_{2} \mathrm{ScHO}_{3}$ (green) and $\mathrm{Ba}_{2} \mathrm{ScHO}_{3}$ (red).

Activation barriers were calculated for the same processes described above for $\mathrm{Ba}_{2} \mathrm{ScHO}_{3}$. Results and a comparison between the two compounds are shown in Figure 5. The transition states, shown in Figure S8, all contain mobile hydride ions in equivalent coordination environments to their $\mathrm{Ba}_{2} \mathrm{ScHO}_{3}$ counterparts. However, the barrier heights between the compounds show significant differences most clearly indicated by the bar chart at the end of Figure 5. The two-atom interstitialcy mechanism remains the lowest energy pathway, with a barrier of $0.19 \mathrm{eV}$, and the 'Along' and 'Across' vacancy hops are very close in energy with barriers of $0.24 \mathrm{eV}$ and $0.26 \mathrm{eV}$ respectively. The interstitial mechanism is then far higher, at $1.04 \mathrm{eV}$. We can compare these results to those for the barium analogue; the three lowest barriers in $\mathrm{Sr}_{2} \mathrm{ScHO}_{3}$, namely the minimum-energy interstitialcy pathway and both 
vacancy hops, have heights approximately half those seen in $\mathrm{Ba}_{2} \mathrm{ScHO}_{3}$, while the interstitial hop is raised slightly. The highest energy pathway makes a minimal contribution to hydride mobility, so the most important result is the dramatic reduction in barriers for interstitialcy migration, which remains the lowest energy pathway, and both modes of vacancy migration. The interstitialcy hop transition state contains mobile hydrides in near-trigonal planar coordination with H-Sr bond lengths of $2.44 \AA(\times 2)$ and $2.41 \AA(\times 1)$. The high-energy interstitial hop TS has the hydride in linear coordination with H-Sr bond lengths of $2.21 \AA$ and two hydrides at $2.35 \AA$, with these short-range electrostatic repulsions again explaining why this pathway has the highest barrier. In the 'Along' hop TS, $\mathrm{H}^{-}$is again displaced along $c$ from the centre of a Sr tetrahedron, giving pairs of $\mathrm{H}$-Sr bond lengths of $2.48 \AA$ and $2.76 \AA$, and in the 'Across' hop TS $\mathrm{H}^{-}$is in linear coordination with $\mathrm{Sr}^{2+}$ at $2.31 \AA$.

The MSD plots in Figure 5(b,c) show the displacements for hydrogen climbing constantly, indicating hydride diffusion, with gradients far steeper than those for $\mathrm{Ba}_{2} \mathrm{ScHO}_{3}$, demonstrating much higher mobility. The estimated diffusion constants for these simulations are $1.3 \times 10^{-9} \mathrm{~m}^{2} \mathrm{~s}^{-1}$ for the interstitial cell and $8.9 \times 10^{-10} \mathrm{~m}^{2} \mathrm{~s}^{-1}$ for the vacancy cell. This orderof-magnitude increase matches the substantial reduction in mobility barriers on substitution of $\mathrm{Sr}$ for $\mathrm{Ba}$ and supports our prediction that $\mathrm{Sr}_{2} \mathrm{ScHO}_{3}$ will show far greater hydride conductivity than $\mathrm{Ba}_{2} \mathrm{ScHO}_{3}$.

The barriers show that an A-site substitution with a smaller cation results, counterintuitively, in a marked increase in hydride mobility. For the purposes of quantitative structural analysis, we note that the hard-sphere ionic radii of Shannon ${ }^{30}$ for $\mathrm{Sr}^{2+}$ and $\mathrm{Ba}^{2+}$ are 1.31 $\AA$ and $1.47 \AA$ respectively, a radius ratio of $89 \%$. The more modern soft-sphere values of Lang and Smith ${ }^{31}$ give the same ratio. We may then use this value as a yardstick against which to measure the changes in the lattice parameters on on cation substitution - as noted above, these are $2 \%$ for $(a, b)$ and $6 \%$ for $c$. We would expect these values to be $11 \%$ if they were governed in a simple way by the ionic radii, so we must examine the structure more closely to understand our observations. Along $c$ the unit cell contains perovskite layers, 
spanned by Sc-O and Sc-H bonds, and rock salt layers, spanned by Sr-O and Sr-H bonds, while $a$ and $b$ are fully spanned both by Sc-O and Sr-O bonds. We can thus understand the non-uniformity in the variation of the cell parameters between $\mathrm{Ba}_{2} \mathrm{ScHO}_{3}$ and $\mathrm{Sr}_{2} \mathrm{ScHO}_{3}$. Along $c$ the Sr-O and Sr-H bonds across the rock salt layers can shorten freely to adopt their optimal length, while changes along $a$ and $b$ are largely prevented by the more rigid Sc-O bonds. This makes the $\mathrm{Sr}-\mathrm{O} / \mathrm{H}$ bonds in the $a b$ plane longer than optimal, so the ions have greater freedom to move in this plane and can more readily accommodate mobile hydride ions than in the $\mathrm{Ba}$ analogue. This can be seen in the transition state geometries, which feature greater displacements of ions surrounding the mobile hydride.

The consequences of greater in-plane structural flexibility are not confined to the rock salt layers as the TS geometries for $\mathrm{Sr}_{2} \mathrm{ScHO}_{3}$ also show small octahedral tilts which were absent in $\mathrm{Ba}_{2} \mathrm{ScHO}_{3}$. This correlates with the trend in Goldschmidt's tolerance factor $t$ - as the radius of the $\mathrm{A}$ cation increases, $t$ falls, suggesting a greater propensity for octahedral tilting. This measure governs cubic perovskites and is therefore only qualitatively applicable to layered oxyhydrides, but it nevertheless captures the relationship between cation size and local distortions which can stabilize transition states.

\section{Discussion}

$\mathrm{Ba}_{2} \mathrm{ScO}_{3} \mathrm{H}$ is of immediate interest in the developing field of hydride conduction because of its unusual anion ordering, which preferentially places hydrides on the lattice sites bordering the rock salt layer in the RP framework. As anticipated, this new ordering enables conduction by pathways as yet unexplored by hydride conductors. ${ }^{17}$ The most important lattice site for hydride migration is the tetrahedral site in the rock salt layer in which an anion is surrounded by four A-site cations; this is the geometry both of the interstitial hydride in its resting state and the transition states for vacancy hops. For many hydride conductors these sites are inaccessible as the hydrides are contained entirely in the octahedral layer. In 
their study of $\mathrm{Ln}_{2} \mathrm{LiHO}_{3}(\mathrm{Ln}=\mathrm{La}, \mathrm{Pr}, \mathrm{Nd})$ Iwasaki et al. ${ }^{32}$ calculated a barrier to interstitial migration through the rock salt layer of $1.4 \mathrm{eV}$. That family of oxyhydrides contains hydride anions in the perovskite layers, so hydrides in the tetrahedral interstitial sites would have no intersticialcy mechanism available to facilitate hydride transport.

The significance of this tetrahedral site is consistent with RP-phase chemistry in the broader field of oxide conduction. Oxygen-rich phases based on $\mathrm{La}_{2} \mathrm{NiO}_{4}$ have been shown to have high anisotropic oxide mobility, ${ }^{9,33}$ arising from conduction through the rock salt layers, and the tetrahedral sites are central to oxide conduction in $\operatorname{Pr}_{2} \mathrm{NiO}_{4+\delta} \cdot{ }^{18}$ Meanwhile, $\mathrm{La}_{2-x} \mathrm{Sr}_{x} \mathrm{CuO}_{4-y}$ shows in-plane vacancy-driven oxide conduction at low strontium concentration. ${ }^{34}$ The tetrahedral sites can also be occupied stoichiometrically, in preference to the "normal" apical site, if cation size constraints require it. This is observed in the series $(\mathrm{La} / \mathrm{Nd})_{2} \mathrm{CuO}_{4}$, where the all-La member has the $\mathrm{K}_{2} \mathrm{NiF}_{4}$ structure, the all-Nd member places all rock-salt-layer oxides in tetrahedral sites, and a 1:1 La:Nd ratio gives alternating behaviour between rock salt layers. ${ }^{35}$

At ambient pressure, the lowest barriers for vacancy and interstitial mobility are approximately equal, at $0.41 \mathrm{eV}$ and $0.35 \mathrm{eV}$ respectively. Assuming other, higher-energy pathways make only a minor contribution to the overall ion mobility, we conclude that both types of defect promote hydride conduction equally. It is also interesting that the overall lowest barrier describes a cooperative motion between two hydrides; a defect-rich phase may have access to cooperative motions involving more atoms, providing new low-energy pathways for long-range hydride movement. Takeiri et al. ${ }^{36}$ showed B-site doping of $\mathrm{SrTiO}_{3}$ by $\mathrm{Sc}^{3+}$ increased the concentration of anion vacancies, so it is plausible that similar strategies could introduce more defects, vacancies or interstitials, into $\mathrm{Ba}_{2} \mathrm{ScHO}_{3}$. We note here that these barriers are somewhat lower than the $0.88 \mathrm{eV}$ measured experimentally; we attribute this difference to possible defect association and the approximations of DFT, but we remain confident in our predicted trends.

Under high pressure, we see significant differences in the behaviour of interstitial and va- 
cancy defects and a change in the dominant conduction mechanism. Due to substantial compression of bond lengths in low-coordinate transition states, the barriers to interstitial defect migration rise far more dramatically under pressure than those to vacancy migration. Hydride conduction at 20 GPa may therefore be accessible if mediated by vacancies, but the structural changes under compression immobilize interstitials.

We investigated the effect of changing the A-site cation by studying the hypothetical compound $\mathrm{Sr}_{2} \mathrm{ScHO}_{3}$, which we believe will show significantly improved hydride conductivity over its barium-containing cousin. The improvement is attributed to greater flexibility in the $a b$ plane because, despite the smaller A-site cations, $a$ and $b$ do not shorten because of the stiffness of the Sc-O bonds. The behaviour is consistent with high-pressure studies on the related phases $\mathrm{SrVO}_{2} \mathrm{H}$ and $\mathrm{Sr}_{2} \mathrm{VO}_{3} \mathrm{H}$ where the V-O bonds resist compression most strongly, leading to anisotropic cell deformation. ${ }^{13,29}$ We therefore see another example here of the behaviour of Ruddlesden-Popper derivatives being dominated by their dimensionality; the infinite layers of Sc-O bonds are integral to giving the $\mathrm{Sr}-\mathrm{H}$ bonds the flexibility needed for improved ion conductivity.

The effect of changing the A-site cation has been investigated in other oxyhydride ion conductors. Very similar behaviour was observed in the cubic perovskites $\mathrm{SrTiO}_{3-x} \mathrm{H}_{x}$ and $\mathrm{BaTiO}_{3-x} \mathrm{H}_{x},{ }^{37,38}$ where the barrier to vacancy migration was calculated as $0.17 \mathrm{eV}$ for the former and $0.28 \mathrm{eV}$ for the latter. These cubic perovskites also contain rigid bonding scaffolds, composed of Ti-O bonds, so it is plausible that this represents a three-dimensional example of the effect of $\mathrm{Sr}-\mathrm{H}$ bond flexibility. This interpretation predicts that conductivity in these perovskite oxyhydrides could be improved further by using a yet smaller cation like $\mathrm{Ca}^{2+}$, or an A-site solid solution if such a small cation drives a transition to a new structure type.

In $\mathrm{Ln}_{2} \mathrm{LiHO}_{3}$ the identity of the lanthanide was found to govern hydride conductivity via changes in anion disorder ${ }^{32}$ - since the hydrides in those phases are contained in the rock salt layers, and thus coordinated principally to the B cations, it is unsurprising that the 
effect of changing the size of the A cation is less direct than in our phases. A similar effect was seen in the LnHO series ${ }^{4}$ in which the mobile hydrides must pass through a trigonal cation environment reminiscent of the transition state for the interstitialcy mechanism in $\mathrm{Ba}_{2} \mathrm{ScHO}_{3}$, where the size of the "bottleneck" expanded significantly when the size of the cation induced $\mathrm{O} / \mathrm{H}$ ordering. The authors also found that a "critical bottleneck radius" of $1.18 \AA$ was required for fast ion conduction - we have calculated these radii for the trigonal sites in our interstitialcy mechanisms and found values of $1.15 \AA$ for $\mathrm{Ba}_{2} \mathrm{ScHO}_{3}$ and 1.19 $\AA$ for $\mathrm{Sr}_{2} \mathrm{ScHO}_{3}$, consistent with our prediction that $\mathrm{Sr}_{2} \mathrm{ScHO}_{3}$ displays significantly higher conductivity.

\section{Summary and conclusions}

We sought to understand hydride conduction in $\mathrm{Ba}_{2} \mathrm{ScO}_{3} \mathrm{H}$, a recently synthesized RuddlesdenPopper oxyhydride with a novel anion ordering. We have identified the most important pathways that give rise to hydride mobility arising from both vacancy and interstitial defects, showing that both types make an approximately equal contribution to conductivity. The anion ordering was found to give rise to conduction pathways previously unknown in oxyhydride chemistry, using interstitial positions known to be important in the corresponding oxide chemistry. AIMD simulations confirm that in this compound the ionic conductivity arises from the hydrides only. We have also shown that the anion ordering pattern in the rock salt layers does not substantially affect the most important barriers, so a compound of $\mathrm{A}_{2} \mathrm{BO}_{2} \mathrm{H}_{2}$ stoichiometry containing only hydrides in the rock salt layers would have higher hydride conduction due to the greater number of labile hydrides but not due to lower activation barriers.

We studied the effect of pressure on the structure and on hydride mobility, finding this compound comparable to other Ruddlesden-Popper oxyhydrides. At $20 \mathrm{GPa}$ the migration barriers for vacancy defects are far lower than those for interstitials, so a vacancy-rich sam- 
ple would have a higher conductivity under pressure than an interstitial-rich sample, the conduction in which would be more pressure-sensitive.

While investigating the consequences of changing the A-site cation, we found that the migration barriers for the most favourable hydride diffusion pathways are significantly lower in $\mathrm{Sr}_{2} \mathrm{ScHO}_{3}$ than in $\mathrm{Ba}_{2} \mathrm{ScHO}_{3}$. We therefore predict that this compound would show improved hydride conductivity, and we hope our work encourages experimental study of these compounds. We believe this to be a result of greater structural flexibility localized in the rock salt layers when the A-site cation is chosen to be smaller but the rigid perovskite layer is unchanged, so we also predict greater conductivity in the calcium analogue or a phase containing a mixture of A-site cations. Such an effect is a unique feature of this family of oxyhydrides since most preferentially contain hydrides in the perovskite layer. All of our observed trends in ionic mobility have been explained by widely-applicable crystal engineering arguments, and we therefore believe that they can be used to guide design of future perovskite ion conductors.

\section{Acknowledgement}

The authors thank John McGrady, Chris Mohn and Jean-Alexis Hernandez for valuable discussions, the EPSRC for support through the Centre for Doctoral Training, Theory and Modelling in Chemical Sciences, under grant EP/L015722/1, the UK Materials and Molecular Modelling Hub for computational resources, which is partially funded by EPSRC (EP/P020194/1), and the Advanced Computing Research Centre at the University of Bristol https://www.bristol.ac.uk/acrc/. HWTM thanks the Radcliffe scholarship fund at University College, Oxford. 


\section{Supporting Information Available}

Supplementary figures as indicated in the text and coordinates of optimized geometries and transition states for $\mathrm{Ba}_{2} \mathrm{ScHO}_{3}$ and $\mathrm{Sr}_{2} \mathrm{ScHO}_{3}$.

\section{References}

(1) Meng, Y. Q.; Gao, J.; Zhao, Z. Y.; Amoroso, J.; Tong, J. H.; Brinkman, K. S. Review: recent progress in low-temperature proton-conducting ceramics. Journal of Materials Science 2019, 54, 9291-9312.

(2) Kim, J.; Sengodan, S.; Kim, S.; Kwon, O.; Bu, Y.; Kim, G. Proton conducting oxides: A review of materials and applications for renewable energy conversion and storage. Renewable 6 Sustainable Energy Reviews 2019, 109, 606-618.

(3) Gao, H.; Lian, K. Proton-conducting polymer electrolytes and their applications in solid supercapacitors: a review. RSC Advances 2014, 4, 33091-33113.

(4) Ubukata, H.; Broux, T.; Takeiri, F.; Shitara, K.; Yamashita, H.; Kuwabara, A.; Kobayashi, G.; Kageyama, H. Hydride Conductivity in an Anion-Ordered Fluorite Structure LnHO with an Enlarged Bottleneck. Chemistry of Materials 2019, 31, 73607366.

(5) Fukui, K.; Iimura, S.; Tada, T.; Fujitsu, S.; Sasase, M.; Tamatsukuri, H.; Honda, T.; Ikeda, K.; Otomo, T.; Hosono, H. Characteristic fast $\mathrm{H}^{-}$ion conduction in oxygensubstituted lanthanum hydride. Nature Communications 2019, 10.

(6) Verbraeken, M. C.; Cheung, C.; Suard, E.; Irvine, J. T. S. High $\mathrm{H}^{-}$ionic conductivity in barium hydride. Nature Materials 2015, 14, 95-100.

(7) Kobayashi, G.; Hinuma, Y.; Matsuoka, S.; Watanabe, A.; Iqbal, M.; Hirayama, M.; 
Yonemura, M.; Kamiyama, T.; Tanaka, I.; Kanno, R. Pure $\mathrm{H}^{-}$conduction in oxyhydrides. Science 2016, 351, 1314-1317.

(8) Jacobson, A. J. Materials for Solid Oxide Fuel Cells. Chemistry of Materials 2010, 22, 660-674.

(9) Bassat, J. M.; Odier, P.; Villesuzanne, A.; Marin, C.; Pouchard, M. Anisotropic ionic transport properties in $\mathrm{La}_{2} \mathrm{NiO}_{4+\delta}$ single crystals. Solid State Ionics 2004, 167, 341347.

(10) Watanabe, A.; Kobayashi, G.; Matsui, N.; Yonemura, M.; Kubota, A.; Suzuki, K.; Hirayama, M.; Kanno, R. Ambient Pressure Synthesis and $\mathrm{H}^{-}$Conductivity of $\mathrm{LaSrLiH}_{2} \mathrm{O}_{2}$. Electrochemistry 2017, 85, 88-92.

(11) Hayward, M. A.; Cussen, E. J.; Claridge, J. B.; Bieringer, M.; Rosseinsky, M. J.; Kiely, C. J.; Blundell, S. J.; Marshall, I. M.; Pratt, F. L. The hydride anion in an extended transition metal oxide array: $\mathrm{LaSrCoO}_{3} \mathrm{H}_{0.7}$. Science 2002, 295, 1882-1884.

(12) Bridges, C. A.; Darling, G. R.; Hayward, M. A.; Rosseinsky, M. J. Electronic structure, magnetic ordering, and formation pathway of the transition metal oxide hydride $\mathrm{LaSrCoO}_{3} \mathrm{H}_{0.7}$. Journal of the American Chemical Society 2005, 127, 5996-6011.

(13) Yamamoto, T.; Morgan, H. W. T.; Zeng, D. H.; Kawakami, T.; Patino, M. A.; Hayward, M. A.; Kageyama, H.; McGrady, J. E. Pressure-Induced Transitions in the 1Dimensional Vanadium Oxyhydrides $\mathrm{Sr}_{2} \mathrm{VO}_{3} \mathrm{H}$ and $\mathrm{Sr}_{3} \mathrm{~V}_{2} \mathrm{O}_{5} \mathrm{H}_{2}$, and Comparison to 2-Dimensional $\mathrm{SrVO}_{2} \mathrm{H}$. Inorganic Chemistry 2019, 58, 15393-15400.

(14) Bai, Q.; He, X. F.; Zhu, Y. Z.; Mo, Y. F. First-Principles Study of Oxyhydride $\mathrm{H}^{-}$Ion Conductors: Toward Facile Anion Conduction in Oxide-Based Materials. ACS Applied Energy Materials 2018, 1, 1626-1634. 
(15) Fjellvåg, O. S.; Armstrong, J.; Vajeeston, P.; Sjåstad, A. O. New Insights into Hydride Bonding, Dynamics, and Migration in $\mathrm{La}_{2} \mathrm{LiHO}_{3}$ Oxyhydride. Journal of Physical Chemistry Letters 2018, 9, 353-358.

(16) Fjellvåg, O. S.; Krzystyniak, M.; Vajeeston, P.; Sjåstad, A. O.; Armstrong, J. A combined deep inelastic neutron scattering and ab initio lattice dynamics study of the hydride anion dynamics and bonding in $\mathrm{La}_{2} \mathrm{LiHO}_{3}$ oxyhydride. Journal of Physics Communications 2019, 3, 7.

(17) Takeiri, F.; Watanabe, A.; Kuwabara, A.; Nawaz, H.; Ayu, N. I. P.; Yonemura, M.; Kanno, R.; Kobayashi, G. $\mathrm{Ba}_{2} \mathrm{ScHO}_{3}: \mathrm{H}^{-}$Conductive Layered Oxyhydride with $\mathrm{H}^{-}$ Site Selectivity. Inorganic Chemistry 2019, 58, 4431-4436.

(18) Yashima, M.; Enoki, M.; Wakita, T.; Ali, R.; Matsushita, Y.; Izumi, F.; Ishihara, T. Structural disorder and diffusional pathway of oxide ions in a doped $\mathrm{Pr}_{2} \mathrm{NiO}_{4}$-based mixed conductor. Journal of the American Chemical Society 2008, 130, 2762-+.

(19) Kresse, G.; Furthmuller, J. Efficient iterative schemes for ab initio total-energy calculations using a plane-wave basis set. Physical Review B 1996, 54, 11169-11186.

(20) Blochl, P. E. Projector Augmented-Wave Method. Physical Review B 1994, 50, 1795317979.

(21) Perdew, J. P.; Burke, K.; Ernzerhof, M. Generalized gradient approximation made simple. Physical Review Letters 1996, 77, 3865-3868.

(22) Sheppard, D.; Xiao, P. H.; Chemelewski, W.; Johnson, D. D.; Henkelman, G. A generalized solid-state nudged elastic band method. Journal of Chemical Physics 2012, 136, 8.

(23) Henkelman, G.; Uberuaga, B. P.; Jonsson, H. A climbing image nudged elastic band 
method for finding saddle points and minimum energy paths. Journal of Chemical Physics 2000, 113, 9901-9904.

(24) Humphrey, W.; Dalke, A.; Schulten, K. VMD: Visual molecular dynamics. Journal of Molecular Graphics 83 Modelling 1996, 14, 33-38.

(25) Nosé, S. A unified formulation of the constant temperature molecular-dynamics methods. Journal of Chemical Physics 1984, 81, 511-519.

(26) Momma, K.; Izumi, F. VESTA: a three-dimensional visualization system for electronic and structural analysis. Journal of Applied Crystallography 2008, 41, 653-658.

(27) Hoover, W. G. Canonical dynamics - equilibrium phase-space distributions. Physical Review A 1985, 31, 1695-1697.

(28) Yamamoto, T.; Kobayashi, Y.; Okada, T.; Yagi, T.; Kawakami, T.; Tassel, C.; Kawasaki, S.; Abe, N.; Niwa, K.; Kikegawa, T.; Hirao, N.; Takano, M.; Kageyama, H. B1-to-B2 Structural Transitions in Rock Salt Intergrowth Structures. Inorganic Chemistry 2011, 50, 11787-11794.

(29) Yamamoto, T.; Zeng, D. H.; Kawakami, T.; Arcisauskaite, V.; Yata, K.; Patino, M. A.; Izumo, N.; McGrady, J. E.; Kageyama, H.; Hayward, M. A. The role of pi-blocking hydride ligands in a pressure-induced insulator-to-metal phase transition in $\mathrm{SrVO}_{2} \mathrm{H}$. Nature Communications 2017, 8 .

(30) Shannon, R. D. Revised effective ionic-radii and systematic studies of interatomic distances in halides and chalcogenides. Acta Crystallographica Section A 1976, 32, 751767.

(31) Lang, P. F.; Smith, B. C. Ionic radii for Group 1 and Group 2 halide, hydride, fluoride, oxide, sulfide, selenide and telluride crystals. Dalton Transactions 2010, 39, 7786-7791. 
(32) Iwasaki, Y.; Matsui, N.; Suzuki, K.; Hinuma, Y.; Yonemura, M.; Kobayashi, G.; Hirayama, M.; Tanaka, I.; Kanno, R. Synthesis, crystal structure, and ionic conductivity of hydride ion-conducting $\mathrm{Ln}_{2} \mathrm{LiHO}_{3}$ ( $\mathrm{Ln}=\mathrm{La}, \mathrm{Pr}, \mathrm{Nd}$ ) oxyhydrides. Journal of Materials Chemistry A 2018, 6, 23457-23463.

(33) Skinner, S. J.; Kilner, J. A. Oxygen diffusion and surface exchange in $\mathrm{La}_{2-\mathrm{x}} \mathrm{Sr}_{\mathrm{x}} \mathrm{NiO}_{4+\delta}$. Solid State Ionics 2000, 135, 709-712.

(34) Opila, E. J.; Tuller, H. L.; Wuensch, B. J.; Maier, J. Oxygen tracer diffusion in $\mathrm{La}_{2-\mathrm{x}} \mathrm{Sr}_{\mathrm{x}} \mathrm{CuO}_{4-\mathrm{y}}$ single crystals. Journal of the American Ceramic Society 1993, 76, $2363-2369$.

(35) Allan, N. L.; Baram, P. S.; Mackrodt, W. C.; Turner, M. J. Differences between highTc oxides containing 6-fold, 5-fold, 4-fold and 2-fold coordinated copper. Molecular Simulation 1992, 9, 115-128.

(36) Takeiri, F.; Aidzu, K.; Yajima, T.; Matsui, T.; Yamamoto, T.; Kobayashi, Y.; Hester, J.; Kageyama, H. Promoted Hydride/Oxide Exchange in $\mathrm{SrTiO}_{3}$ by Introduction of Anion Vacancy via Aliovalent Cation Substitution. Inorganic Chemistry 2017, 56, 1303513040.

(37) Liu, X.; Bjorheim, T. S.; Vines, L.; Fjellvåg, O. S.; Granerod, C.; Prytz, O.; Yamamoto, T.; Kageyama, H.; Norby, T.; Haugsrud, R. Highly Correlated Hydride Ion Tracer Diffusion in $\mathrm{SrTiO}_{3-\mathrm{x}} \mathrm{H}_{\mathrm{x}}$ Oxyhydrides. Journal of the American Chemical Society 2019, 141, 4653-4659.

(38) Liu, X.; Bjorheim, T. S.; Haugsrud, R. Formation and migration of hydride ions in $\mathrm{BaTiO}_{3-\mathrm{x}} \mathrm{H}_{\mathrm{x}}$ oxyhydride. Journal of Materials Chemistry A 2017, 5, 1050-1056. 


\section{Graphical TOC Entry}

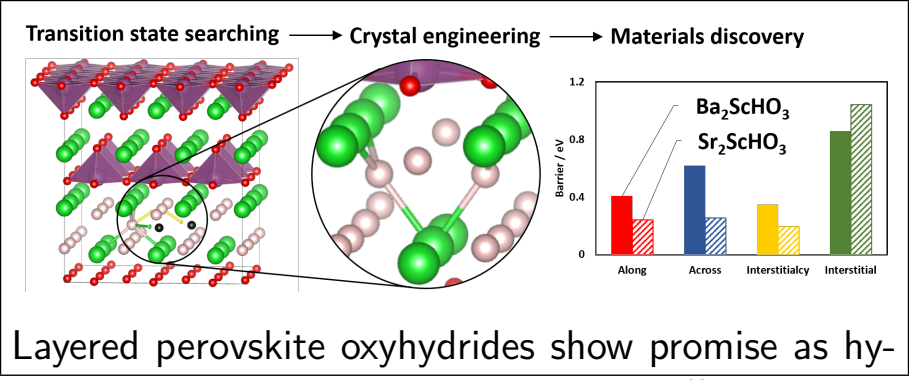

dride ion conductors, but their ionic diffusion pathways are not yet well understood. In this DFT investigation we identify the dominant mechanisms in $\mathrm{Ba}_{2} \mathrm{ScHO}_{3}$, which has an unusual anion ordering, and explain trends by geometrical analysis. On the basis

of our insights, we predict that $\mathrm{Sr}_{2} \mathrm{ScHO}_{3}$ will show improved ionic conductivity. 